\title{
Activities of daily living: a comparison between patients with COPD and ILD
}

\author{
Personalised medicine, COPD - management, Extrapulmonary impact
}

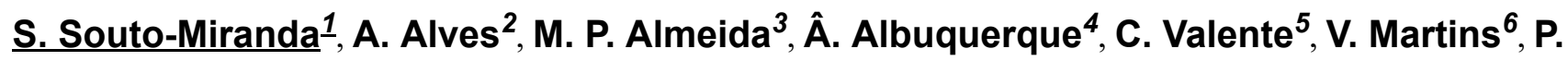
Ferreira $^{5}$, A. Marques ${ }^{7}$

${ }^{1}$ Lab3R - Respiratory Research and Rehabilitation Laboratory, School of Health Sciences, University of Aveiro, Aveiro Portugal; iBiMED - Institute of Biomedicine, School of Health Sciences, University of Aveiro, Aveiro, Portugal - Aveiro (Portugal), ${ }^{2}$ REMEO Home, Linde Healthcare, Portugal - Algarve (Portugal), ${ }^{3}$ School of Health Sciences of the University of Aveiro, Aveiro, Portugal; North Rehabilitation Center, Centro Hospitalar Vila Nova de Gaia, Vila Nova de Gaia, Portugal - Gaia (Portugal), ${ }^{4}$ Department of Pulmonology, Pulmonary Rehabilitation Sector, Hospital Sousa Martins Unidade Local de Saúde da Guarda, Guarda, Portugal - Guarda (Portugal), ${ }^{5}$ Department of Pulmonology, Centro Hospitalar do Vouga, E.P.E, Aveiro, Portugal - Aveiro (Portugal), ${ }^{6}$ Department of Pulmonology, Hospital Distrital da Figueira da Foz, Figueira da Foz, Portugal - Figueira da Foz (Portugal), ${ }^{7}$ Lab3R - Respiratory Research and Rehabilitation Laboratory, School of Health Sciences, University of Aveiro, Aveiro Portugal; iBiMED - Institute of Biomedicine, School of Health Sciences, University of Aveiro, Aveiro, Portugal - Aveiro; School of Health Sciences of the University of Aveiro, Aveiro, Portugal (Portugal)

Due to similarities in extrapulmonary features, patients with chronic obstructive pulmonary disease (COPD) and interstitial lung diseases (ILD) are commonly treated with a one size fits all approach in pulmonary rehabilitation (PR). Though this approach is inadequate, differences between populations in meaningful outcomes, such as activities of daily living (ADL), have been scarcely explored. This study explored the functional performance of patients with COPD and ILD in upper and lower-limb ADL.

Patients with COPD and ILD entering a 12-week community-based PR programme were comprehensively assessed. Functional performance was assessed with Glittre, 1-min sit to stand (STS), grocery shelving task (GST), physical performance (PPT), Chester step and 6-minute walk tests. Data were analysed with Mann-Whitney $U$ tests.

Patients with COPD ( $n=68,72 \%$ male, 66 $\pm 10 y$; FEV1 56 $\pm 20 p p$ ), showed worse functional performance than patients with ILD ( $n=78 ; 46 \%$ male, $65 \pm 14$ years, FVC $77 \pm 19$ DLCO $57 \pm 52 \%$ predicted) in the number of laps in Glittre (COPD 3.9 \pm 1.5 ; ILD 4.6 $\pm 0.9 ; \mathrm{p}=0.014$ ), time of the GST (COPD 49.8 \pm 14.1 ; ILD $45.4 \pm 14.6$; $p=0.018$ ) and score of the PPT (COPD 28.1 \pm 5.6 ; ILD 30.6 $\pm 4.0 ; p=0.008)$. No other significant differences were found.

Significant differences exist in functional performance of ADL that involve the upper limbs between patients with COPD and ILD. Future research is needed to confirm these findings and move PR to a personalised approach. 
Table 1. Data from functional performance measures in patients with COPD and ILD

\section{Functional performance outcome measures}

\begin{tabular}{|c|c|c|c|}
\hline & COPD $(n=33)$ & ILD (n=36) & P-value \\
\hline Glittre, laps & $3.9 \pm 1.5$ & $4.6 \pm 0.9$ & $0.014^{*}$ \\
\hline \multirow[t]{2}{*}{ Glittre, time (s) } & $4.7 \pm 7.9$ & $3.2 \pm 1.3$ & 0.520 \\
\hline & COPD $(n=67)$ & ILD ( $n=76)$ & \\
\hline \multirow[t]{2}{*}{ 1-min STS, repetitions } & $25.9 \pm 10.4$ & $23.6 \pm 8.1$ & 0.244 \\
\hline & COPD $(n=67)$ & ILD (n=63) & \\
\hline \multirow[t]{2}{*}{ Grocery shelving task, time (s) } & $49.8 \pm 14.1$ & $45.4 \pm 14.6$ & $0.018^{*}$ \\
\hline & COPD $(n=59)$ & ILD (n=63) & \\
\hline \multirow{2}{*}{$\begin{array}{l}\text { Physical performance test, } \\
\text { points }\end{array}$} & $28.1 \pm 5.6$ & $30.6 \pm 4.0$ & $0.008^{*}$ \\
\hline & COPD $(n=49)$ & ILD $(n=70)$ & \\
\hline \multirow[t]{2}{*}{ Chester step test, steps } & $80.6 \pm 62.9$ & $80.3 \pm 54.0$ & 0.646 \\
\hline & COPD $(n=68)$ & ILD ( $n=78)$ & \\
\hline 6MWT, m & $404.4 \pm 113.0$ & $407.9 \pm 134.9$ & 0.900 \\
\hline
\end{tabular}

Data is shown as mean $\pm S D$.

STS: Sit to stand; 6MWT: 6-minute walk test 